September 18, 2021

\title{
PROPER AND PIECEWISE PROPER FAMILIES OF REALS
}

\author{
VICTORIA GITMAN
}

\begin{abstract}
I introduced the notions of proper and piecewise proper families of reals to make progress on a long standing open question in the field of models of Peano Arithmetic about whether every Scott set is the standard system of a model of PA. A Scott set is a family of reals closed under $\Delta_{1}$ definability and satisfying weak Konig's Lemma. A family of reals $\mathfrak{X}$ is proper if it is arithmetically closed and the quotient Boolean algebra $\mathfrak{X} /$ Fin is a proper partial order. A family is piecewise proper if it is the union of a chain of proper families of size $\leq \omega_{1}$. I showed that under the Proper Forcing Axiom, every proper or piecewise proper family of reals is the standard system of a model of PA. Here, I explore the question of the existence of proper and piecewise proper families of reals of different cardinalities.
\end{abstract}

\section{INTRODUCTION}

One of the central concepts in the field of models of Peano Arithmetic is the standard system of a model of PA. The standard system of a model of PA is the collection of subsets of the natural numbers that arise as intersections of the definable sets of the model with its standard part $\mathbb{N}$. The notion of a $S$ cott set captures three key properties of standard systems.

Definition 1.1. $\mathfrak{X} \subseteq \mathcal{P}(\mathbb{N})$ is a Scott set if

(1) $\mathfrak{X}$ is a Boolean algebra of sets.

(2) If $A \in \mathfrak{X}$ and $B$ is Turing computable from $A$, then $B \in \mathfrak{X}$.

(3) If $T$ is an infinite binary tree coded by a set in $\mathfrak{X}$, then $\mathfrak{X}$ has a set coding some path through $T$.

In 1962, Scott showed that every standard system is a Scott set and the partial converse that every countable Scott set is the standard system of a model of PA [?]. The question of whether every Scott is the standard system of a model of PA became known in the folklore as Scott's Problem. In 1982, Knight and Nadel extended Scott's result to Scott sets of size $\omega_{1}$ [?]. They showed that every Scott set of size $\omega_{1}$ is the standard system of a model of PA. It has proved very difficult to make further progress on Scott's Problem. My approach, following Engström [?] and suggested several years earlier by Hamkins, Marker, etc., has been to use the set theoretic techniques of forcing and the forcing axioms.

We can associate with every family of reals $\mathfrak{X}$, the poset $\mathfrak{X} /$ Fin which consists of the infinite sets of $\mathfrak{X}$ under the ordering of almost inclusion. Engström in [?] introduced the use of this poset in connection with Scott's Problem. A family of reals is arithmetically closed if whenever $A$ is in it and $B$ is arithmetically definable from $A$, then $B$ is also in it. The arithmetic closure of $\mathfrak{X}$ is an essential ingredient in the constructions that make the posets $\mathfrak{X} /$ Fin useful in investigating properties of uncountable models of PA (for details of the constructions, see [?]). For this reason, whenever we view a family of reals as a poset we will always assume arithmetic 
closure. A family $\mathfrak{X}$ is proper if it is arithmetically closed and the poset $\mathfrak{X} /$ Fin is proper. A family $\mathfrak{X}$ is piecewise proper if it is the union of a chain of proper families each of which has size $\leq \omega_{1}$. I showed in [?] that under the Proper Forcing Axiom (PFA), every proper or piecewise proper family of reals is the standard system of a model of PA. I will give an extended discussion of properness and the PFA in Section 2

Throughout the paper, I equate reals with subsets of $\mathbb{N}$. It is easy to see that every countable arithmetically closed family of reals is proper and $\mathcal{P}(\mathbb{N})$ is proper as well (see Section 3). Every arithmetically closed family of size $\leq \omega_{1}$ is trivially piecewise proper since it is the union of a chain of countable arithmetically closed families. It becomes much more difficult to find instances of uncountable proper families of reals other than $\mathcal{P}(\mathbb{N})$. Also, it was not clear for a while whether there are piecewise proper families of of size larger than $\omega_{1}$. My main results are:

Theorem 1.2. If $\mathrm{CH}$ holds, then $\mathcal{P}^{V}(\mathbb{N}) /$ Fin remains proper in any generic extension by a c.c.c. poset.

Theorem 1.3. There is a generic extension of $V$ by a c.c.c. poset, which contains continuum many proper families of reals of size $\omega_{1}$.

Theorem 1.4. There is a generic extension of $V$ by a c.c.c. poset, which contains continuum many piecewise proper families of reals of size $\omega_{2}$.

\section{Proper Posets And the PFA}

Proper posets were invented by Shelah, who sought a class of $\omega_{1}$ preserving posets that would extend the c.c.c. and countably closed classes of posets and be preserved under iterations with countable support. The Proper Forcing Axiom (PFA) was introduced by Baumgartner who showed that it is consistent by assuming the existence of a supercompact cardinal [?]. This remains the best known upper bound on the consistency of PFA.

Recall that for a cardinal $\lambda$, the set $H_{\lambda}$ is the collection of all sets whose transitive closure has size less than $\lambda$. Let $\mathbb{P}$ be a poset and $\lambda$ be a cardinal greater than $2^{|\mathbb{P}|}$. Since we can always take an isomorphic copy of $\mathbb{P}$ on the cardinal $|\mathbb{P}|$, we can assume without loss of generality that $\mathbb{P}$ and $\mathcal{P}(\mathbb{P})$ are elements of $H_{\lambda}$. In particular, we want to ensure that all dense subsets of $\mathbb{P}$ are in $H_{\lambda}$. Let $M$ be a countable elementary submodel of $H_{\lambda}$ containing $\mathbb{P}$ as an element. If $G$ is a filter on $\mathbb{P}$, we say that $G$ is $M$-generic if for every maximal antichain $A \in M$ of $\mathbb{P}$, the intersection $G \cap A \cap M \neq \emptyset$. It must be explicitly specified what $M$-generic means in this context since the usual notion of generic filters makes sense only for transitive structures and $M$ is not necessarily transitive. This definition of $M$-generic is closely related to the definition for transitive structures. To see this, let $M^{*}$ be the Mostowski collapse of $M$ and $\mathbb{P}^{*}$ be the image of $\mathbb{P}$ under the collapse. Let $G^{*} \subseteq \mathbb{P}^{*}$ be the pointwise image of $G \cap M$ under the collapse. Then $G$ is $M$-generic if and only if $G^{*}$ is $M^{*}$-generic for $\mathbb{P}^{*}$ in the usual sense.

Later we will need the following important characterization of $M$-generic filters.

Theorem 2.1. If $\mathbb{P}$ is a poset in $M \prec H_{\lambda}$, then a $V$-generic filter $G \subseteq \mathbb{P}$ is $M$-generic if and only if $M \cap \operatorname{Ord}=M[G] \cap$ Ord. (See, for example, [?], p. 105)

Definition 2.2. Let $\mathbb{P} \in H_{\lambda}$ be a poset and $M$ be an elementary submodel of $H_{\lambda}$ containing $\mathbb{P}$. Then a condition $q \in \mathbb{P}$ is $M$-generic if and only if every $V$-generic filter $G \subseteq \mathbb{P}$ containing $q$ is $M$-generic. 
Definition 2.3. A poset $\mathbb{P}$ is proper if for every $\lambda>2^{|\mathbb{P}|}$ and every countable $M \prec H_{\lambda}$ containing $\mathbb{P}$, for every $p \in \mathbb{P} \cap M$, there is an $M$-generic condition below $p$.

When proving that a poset is proper it is often easier to use the following equivalent characterization which appears in [?] (p. 102).

Theorem 2.4. A poset $\mathbb{P}$ is proper if there exists a $\lambda>2^{|\mathbb{P}|}$ and a club of countable $M \prec H_{\lambda}$ containing $\mathbb{P}$, such that for every $p \in \mathbb{P} \cap M$, there is an $M$-generic condition below $p$.

Countably closed posets and c.c.c. posets are proper and all proper posets preserve $\omega_{1}[?]$.

Definition 2.5. The Proper Forcing Axiom (PFA) is the assertion that for every proper poset $\mathbb{P}$ and every collection $\mathcal{D}$ of at most $\omega_{1}$ many dense subsets of $\mathbb{P}$, there is a filter on $\mathbb{P}$ that meets all of them.

The Proper Forcing Axiom decides the size of the continuum. It was shown in [?] that under PFA, continuum is $\omega_{2}$.

\section{Proper and Piecewise Proper Families}

Let $\mathfrak{X}$ be a family of reals. Define the poset $\mathfrak{X} /$ Fin to consist of the infinite sets in $\mathfrak{X}$ under the ordering of almost inclusion. That is, for infinite $A$ and $B$ in $\mathfrak{X}$, we say that $A \leq B$ if and only if $A \subseteq_{\text {Fin }} B$. Observe that $\mathfrak{X} /$ Fin is forcing equivalent to forcing with the Boolean algebra $\mathfrak{X}$ modulo the ideal of finite sets. A familiar and thoroughly studied instance of this poset is $\mathcal{P}(\omega) /$ Fin. For a property of posets $\mathscr{P}$, if $\mathfrak{X}$ is an arithmetically closed family of reals and $\mathfrak{X} /$ Fin has $\mathscr{P}$, I will simply say that $\mathfrak{X}$ has property $\mathscr{P}$. An important point to be noted here is that whenever a family $\mathfrak{X}$ is discussed as a poset, I will always be assuming that it is arithmetically closed. Recall that the reason for this is the need for arithmetic closure of $\mathfrak{X}$ in the constructions with models of PA in which $\mathfrak{X} /$ Fin is used.

The easiest way to show that a poset is proper to show that it is c.c.c. or countably closed. Thus, every countable arithmetically closed family is proper since it is c.c.c. and $\mathcal{P}(\mathbb{N})$ is proper since it is countably closed. Unfortunately these two conditions do not give us any other instances of proper families.

Theorem 3.1. Every c.c.c. family of reals is countable.

Proof. Let $\mathfrak{X}$ be an arithmetically closed family of reals. If $x$ is a finite subset of $\mathbb{N}$, let $\ulcorner x\urcorner$ denote the code of $x$ using Gödel's coding. For every $A \in \mathfrak{X}$, define an associated $A^{\prime}=\{\ulcorner A \cap n\urcorner \mid n \in \mathbb{N}\}$. Clearly $A^{\prime}$ is definable from $A$, and hence in $\mathfrak{X}$. Observe that if $A \neq B$, then $\left|A^{\prime} \cap B^{\prime}\right|<\omega$. Hence if $A \neq B$, we get that $A^{\prime}$ and $B^{\prime}$ are incompatible in $\mathfrak{X} /$ Fin. It follows that $\mathscr{A}=\left\{A^{\prime} \mid A \in \mathfrak{X}\right\}$ is an antichain of $\mathfrak{X} /$ Fin of size $|\mathfrak{X}|$. This shows that $\mathfrak{X} /$ Fin always has antichains as large as the whole poset.

Thus, the poset $\mathfrak{X} /$ Fin has the worst possible chain condition, namely $|\mathfrak{X}|^{+}$-c.c..

Theorem 3.2. Every countably closed family of reals is $\mathcal{P}(\mathbb{N})$.

Proof. I will show that every $A \subseteq \mathbb{N}$ is in $\mathfrak{X}$. Define a sequence of subsets $\left\langle B_{n}\right| n \in$ $\omega\rangle$ by $B_{n}=\left\{m \in \mathbb{N} \mid(m)_{n}=\chi_{A}(n)\right\}$ where $\chi_{A}$ is the characteristic function of $A$. Let $A_{m}=\cap_{n \leq m} B_{n}$ and observe that each $A_{m}$ is infinite. Thus, $A_{0} \geq A_{1} \geq \cdots \geq$ 
$A_{m} \geq \ldots$ are elements of $\mathfrak{X} /$ Fin. By countable closure, there exists $C \in \mathfrak{X} /$ Fin such that $C \subseteq_{\text {Fin }} A_{m}$ for all $m \in \mathbb{N}$. Thus, $C \subseteq_{\text {Fin }} B_{n}$ for all $n \in \mathbb{N}$. It follows that $A=\left\{n \in \mathbb{N} \mid \exists m \forall k \in C\right.$ if $k>m$, then $\left.(k)_{n}=1\right\}$. This shows that $A$ is arithmetic in $C$, and hence $A \in \mathfrak{X}$ by arithmetic closure. Since $A$ was arbitrary, this concludes the proof that $\mathfrak{X}=\mathcal{P}(\mathbb{N})$.

The assumption that $\mathfrak{X}$ is arithmetically closed is not necessary for Theorem 3.2 Any family of reals $\mathfrak{X}$ such that $\mathfrak{X} /$ Fin is countably closed must be arithmetically closed (see [?]) .

Enayat showed in [?] that ZFC proves the existence of an arithmetically closed family of size $\omega_{1}$ which collapses $\omega_{1}$, and hence is not proper.

Later Enayat and Shelah showed in [?] that there is a Borel arithmetically closed family of size $\omega_{1}$ which is not proper as well.

I will show below that it is consistent with ZFC that there are continuum many proper families of size $\omega_{1}$ and it is consistent with ZFC that there are continuum many piecewise proper families of size $\omega_{2}$. But first I will consider the question of when does forcing to add new reals preserve the properness of the reals of the ground model. I will show that if $\mathrm{CH}$ holds, forcing with a c.c.c. poset preserves the properness of the reals of the ground model.

Lemma 3.3. Let $\mathfrak{X}_{0} \subseteq \mathfrak{X}_{1} \subseteq \cdots \subseteq \mathfrak{X}_{\xi} \subseteq \cdots$ for $\xi<\omega_{1}$ be a continuous chain of countable families of reals and let $\mathfrak{X}=\cup_{\xi<\omega_{1}} \mathfrak{X}_{\xi}$. If $M$ is a countable elementary substructure of some $H_{\lambda}$ and $\left\langle\mathfrak{X}_{\xi} \mid \xi<\omega_{1}\right\rangle \in M$, then $M \cap \mathfrak{X}=\mathfrak{X}_{\alpha}$ where $\alpha=$ $\operatorname{Ord}^{M} \cap \omega_{1}$.

Proof. Let $\alpha=\operatorname{Ord}^{M} \cap \omega_{1}$. Suppose $\xi \in \alpha$, then $\xi \in M$, and hence $\mathfrak{X}_{\xi} \in M$. Since $\mathfrak{X}_{\xi}$ is countable, it follows that $\mathfrak{X}_{\xi} \subseteq M$. Thus, $\mathfrak{X}_{\alpha} \subseteq \mathfrak{X} \cap M$. Now suppose $A \in \mathfrak{X} \cap M$, then the least $\xi$ such that $A \in \mathfrak{X}_{\xi}$ is definable in $H_{\lambda}$. It follows that $\xi \in M$, and hence $\xi \in \alpha$. Thus, $\mathfrak{X} \cap M \subseteq \mathfrak{X}_{\alpha}$.

Lemma 3.4. Suppose $\mathbb{P}$ is a c.c.c. poset and $G \subseteq \mathbb{Q}$ is $V$-generic for a countably closed poset $\mathbb{Q}$. Then $\mathbb{P}$ remains c.c.c. in $V[G]$.

Proof. Suppose $\mathbb{P}$ does not remain c.c.c. in $V[G]$. Fix a $\mathbb{Q}$-name $\dot{A}$ and $r \in \mathbb{Q}$ such that $r \Vdash$ " $\dot{A}$ is a maximal antichain of $\check{\mathbb{P}}$ of size $\omega_{1}$ ". Choose $q_{0} \leq r$ and $a_{0} \in \mathbb{P}$ such that $q_{0} \Vdash \check{a_{0}} \in \dot{A}$. Suppose that we have defined $q_{0} \geq q_{1} \geq \cdots \geq q_{\xi} \geq \cdots$ for $\xi<\beta$ where $\beta$ is some countable ordinal, together with a corresponding sequence $\left\langle a_{\xi} \mid \xi<\beta\right\rangle$ of elements of $\mathbb{P}$ such that $q_{\xi} \Vdash \check{a}_{\xi} \in \dot{A}$ and $a_{\xi_{1}} \neq a_{\xi_{2}}$ for all $\xi_{1}<\xi_{2}$. By countable closure of $\mathbb{Q}$, we can find $q \in \mathbb{Q}$ such that $q \leq q_{\xi}$ for all $\xi<\beta$. Let $q_{\beta} \leq q$ and $a_{\beta} \in \mathbb{P}$ such that $q_{\beta} \Vdash \check{a}_{\beta} \in \dot{A}$ and $a_{\beta} \neq a_{\xi}$ for all $\xi<\beta$. Such $a_{\beta}$ must exist since we assumed $r \Vdash$ " $\dot{A}$ is a maximal antichain of $\check{\mathbb{Q}}$ of size $\omega_{1}$ " and $q \leq r$. Thus, we can build a descending sequence $\left\langle q_{\xi} \mid \xi<\omega_{1}\right\rangle$ of elements of $\mathbb{Q}$ and a corresponding sequence $\left\langle a_{\xi} \mid \xi<\omega_{1}\right\rangle$ of elements of $\mathbb{P}$ such that $q_{\xi} \Vdash \check{a}_{\xi} \in \dot{A}$. But clearly $\left\langle a_{\xi} \mid \xi<\omega_{1}\right\rangle$ is an antichain in $V$ of size $\omega_{1}$, which contradicts the assumption that $\mathbb{P}$ was c.c.c..

Theorem 3.5. If $\mathrm{CH}$ holds, then $\mathcal{P}^{V}(\mathbb{N}) /$ Fin remains proper in any generic extension by a c.c.c. poset.

Proof. Let $\mathbb{P}$ be a c.c.c. poset and fix a $V$-generic $g \subseteq \mathbb{P}$. In $V$, let $\mathcal{P}(\mathbb{N})=\cup_{\xi<\omega_{1}} \mathfrak{X}_{\xi}$ where each $\mathfrak{X}_{\xi}$ is countable and $\mathfrak{X}_{0} \subseteq \mathfrak{X}_{1} \subseteq \cdots \subseteq \mathfrak{X}_{\xi} \subseteq \cdots$ is a continuous 
chain. For sufficiently large cardinals $\lambda$, it is easy to see that $H_{\lambda}^{V[g]}=H_{\lambda}[g]$. The countable elementary substructures of $H_{\lambda}[g]$ of the form $M[g]$ where $M \subseteq V$ and $\left\langle\mathfrak{X}_{\xi} \mid \xi<\omega_{1}\right\rangle, \mathbb{P} \in M[g]$ form a club. So by Theorem 2.4 it suffices to find generic conditions only for such elementary substructures. Fix a countable $M[g] \prec H_{\lambda}[g]$ in $V[g]$ such that $\left\langle\mathfrak{X}_{\xi} \mid \xi<\omega_{1}\right\rangle, \mathbb{P} \in M[g]$ and $M \subseteq V$. We need to prove that for every $B \in M[g] \cap \mathcal{P}(\mathbb{N})^{V} /$ Fin, there exists $A \in \mathcal{P}(\mathbb{N})^{V} /$ Fin such that $A \subseteq$ Fin $B$ and $A$ is $M[g]$-generic in $V[g]$. By Lemma 3.3 $M[g] \cap \mathcal{P}^{V}(\mathbb{N})=\mathfrak{X}_{\alpha}$ where $\alpha=\operatorname{Ord}^{M} \cap \omega_{1}$. Let $\mathcal{D}=\left\{\mathscr{D} \cap \mathfrak{X}_{\alpha} \mid \mathscr{D} \in M\right.$ and $\mathscr{D}$ dense in $\mathcal{P}^{V}(\mathbb{N}) /$ Fin $\}$. Observe that $\mathcal{D} \subseteq V$ and $|\mathcal{D}|=\omega$. Since $\mathbb{P}$ is c.c.c., we can show that there is $\mathcal{D}^{\prime} \supseteq \mathcal{D}$ of size $\omega$ in $V$. In $V$, use $\mathcal{D}^{\prime}$ and $\mathfrak{X}_{\alpha}$ to define $\mathcal{E}=\left\{\mathscr{D} \in \mathcal{D}^{\prime} \mid \mathscr{D}\right.$ dense in $\left.\mathfrak{X}_{\alpha}\right\}$. It is clear that $\mathcal{D} \subseteq \mathcal{E}$. By the countable closure of $\mathcal{P}(\mathbb{N})^{V} /$ Fin in $V$, we can find an infinite $A \subseteq$ Fin $B$ such that every $\mathscr{D} \in \mathcal{E}$ contains some $C$ above $A$. It follows that $A$ is $M$-generic. In fact, I will show that $A$ is $M[g]$-generic. To verify this, we need to check that whenever $A \in G$ and $G \subseteq \mathcal{P}(\mathbb{N})^{V} /$ Fin is $V[g]$-generic, then $M[g][G] \cap$ Ord $=M[g] \cap$ Ord. Since we are forcing with $\mathbb{P} \times \mathcal{P}(\mathbb{N}) /$ Fin, we have $M[g][G]=M[G][g]$. It is clear that $M \cap$ Ord $=M[g] \cap$ Ord, and so it remains to show that $M[G][g] \cap$ Ord $=M \cap$ Ord. Since $A \in G$ and $A$ is $M$-generic, we have that $M[G] \cap \operatorname{Ord}=M \cap$ Ord. The poset $\mathbb{P}$ remains c.c.c. in $V[G]$ by Lemma 3.4 since $\mathcal{P}(\mathbb{N})^{V} /$ Fin is countably closed. Also we have $M[G] \prec H_{\lambda}[G]$, even though $M[G]$ itself may not be an element of $V[G]$. Let $\mathscr{A}$ be a maximal antichain of $\mathbb{P}$ in $M[G]$, then $\mathscr{A} \in H_{\lambda}[G]$, and hence $\mathscr{A}$ has size $\omega$. It follows that $\mathscr{A} \subseteq M[G]$. Since $g$ is $V[G]$-generic, it must meet $\mathscr{A}$. So $g$ is $M[G]$-generic, and hence $M[G][g] \cap \operatorname{Ord}=M[G] \cap$ Ord.

It follows that it is consistent that there are uncountable proper families other than $\mathcal{P}(\mathbb{N})$. Start in any universe satisfying $\mathrm{CH}$ and force to add a Cohen real. In the resulting generic extension, the reals of $V$ will be an uncountable proper family.

Next, I will show how to force the existence of many proper families. I will begin by looking at what properness translates into in this specific context.

Proposition 3.6. Suppose $\mathfrak{X}$ is a family of reals and $\mathscr{A}$ is a countable antichain of $\mathfrak{X} /$ Fin. Then for $B \in \mathfrak{X}$ :

(1) Every $V$-generic filter $G \subseteq \mathfrak{X} /$ Fin containing $B$ meets $\mathscr{A}$.

(2) There exists a finite list $A_{0}, \ldots, A_{n} \in \mathscr{A}$ such that $B \subseteq_{\text {Fin }} A_{0} \cup \ldots \cup A_{n}$.

Proof.

$(2) \Longrightarrow(1)$ : Suppose $B \subseteq_{\text {Fin }} A_{0} \cup \ldots \cup A_{n}$ for some $A_{0}, \ldots, A_{n} \in \mathscr{A}$. Since a $V$ generic filter $G$ is an ultrafilter, one of the $A_{i}$ must be in $G$.

$(1) \Longrightarrow(2)$ : Assume that every $V$-generic filter $G$ containing $B$ meets $\mathscr{A}$ and suppose toward a contradiction that (2) does not hold. Enumerate $\mathscr{A}=\left\{A_{0}, A_{1}, \ldots, A_{n}, \ldots\right\}$. It follows that for all $n \in \mathbb{N}$, the intersection $B \cap\left(\mathbb{N}-A_{0}\right) \cap \cdots \cap\left(\mathbb{N}-A_{n}\right)$ is infinite. Define $C=\left\{c_{n} \mid n \in \mathbb{N}\right\}$ such that $c_{0}$ is the least element of $B \cap\left(\mathbb{N}-A_{0}\right)$ and $c_{n+1}$ is the least element of $B \cap\left(\mathbb{N}-A_{0}\right) \cap \cdots \cap\left(\mathbb{N}-A_{n+1}\right)$ greater than $c_{n}$. Clearly $C \subseteq B$ and $C \subseteq$ Fin $\left(\mathbb{N}-A_{n}\right)$ for all $n \in \mathbb{N}$. Let $G$ be a $V$-generic filter containing $C$, then $B \in G$ and $\left(\mathbb{N}-A_{n}\right) \in G$ for all $n \in \mathbb{N}$. But this contradicts our assumption that $G$ meets $\mathscr{A}$.

Corollary 3.7. A family of reals $\mathfrak{X}$ is proper if and only if there exists $\lambda>2^{|\mathfrak{X}|}$ such that for every countable $M \prec H_{\lambda}$ containing $\mathfrak{X}$, whenever $C \in M \cap \mathfrak{X} /$ Fin, then there is $B \subseteq_{\text {Fin }} C$ in $\mathfrak{X} /$ Fin such that for every maximal antichain $\mathscr{A} \in M$ of $\mathfrak{X} /$ Fin, there are $A_{0}, \ldots, A_{n} \in \mathscr{A} \cap M$ with $B \subseteq_{\text {Fin }} A_{0} \cup \cdots \cup A_{n}$. 
Proof.

$(\Longrightarrow)$ : Suppose $\mathfrak{X}$ is proper. Then there is $\lambda>2^{|\mathfrak{X}|}$ such that for every countable $M \prec H_{\lambda}$ containing $\mathfrak{X}$ and every $C \in M \cap \mathfrak{X} /$ Fin, there is an $M$-generic $B \subseteq_{\text {Fin }} C$ in $\mathfrak{X} /$ Fin. Fix a countable $M \prec H_{\lambda}$ containing $\mathfrak{X}$ and $C \in M \cap \mathfrak{X} /$ Fin. Let $B \subseteq_{\text {Fin }} C$ be $M$-generic. Thus, every $V$-generic filter containing $B$ must meet $\mathscr{A} \cap M$ for every maximal antichain $\mathscr{A} \in M$ of $\mathfrak{X} /$ Fin. But since $\mathscr{A} \cap M$ is countable, by Proposition 3.6 there exist $A_{0}, \ldots, A_{n} \in \mathscr{A} \cap M$ such that $B \subseteq_{\text {Fin }} A_{0} \cup \cdots \cup A_{n}$. $(\Longleftarrow)$ : Suppose that there is $\lambda>2^{|\mathfrak{X}|}$ such that for every countable $M \prec H_{\lambda}$ containing $\mathfrak{X}$, whenever $C \in M \cap \mathfrak{X} /$ Fin, then there is $B \subseteq_{\text {Fin }} C$ in $\mathfrak{X} /$ Fin such that for every maximal antichain $\mathscr{A} \in M$ of $\mathfrak{X} /$ Fin, there are $A_{0}, \ldots, A_{n} \in \mathscr{A} \cap M$ with $B \subseteq_{\text {Fin }} A_{0} \cup \cdots \cup A_{n}$. Fix a countable $M \prec H_{\lambda}$ with $\mathfrak{X} \in M$ and $C \in M \cap \mathfrak{X} /$ Fin. Let $B \subseteq_{\text {Fin }} C$ be as above. By Proposition 3.6, every $V$-generic filter $G$ containing $B$ must meet $\mathscr{A} \cap M$ for every maximal antichain $\mathscr{A} \in M$. Thus, $B$ is $M$-generic. Since $M$ was arbitrary, we can conclude that $\mathfrak{X}$ is proper.

The hypothesis of Corollary 3.7 can be weakened, by Theorem 2.4, to finding for some $H_{\lambda}$, only a club of countable $M$ having the desired property.

The next definition is key to all the remaining arguments in the paper.

Definition 3.8. Let $\mathfrak{X}$ be a countable family of reals, let $\mathcal{D}$ be some collection of dense subsets of $\mathfrak{X} / F i n$, and let $B \in \mathfrak{X}$. We say that an infinite set $A \subseteq \mathbb{N}$ is $\langle\mathfrak{X}, \mathcal{D}\rangle$-generic below $B$ if $A \subseteq_{\text {Fin }} B$ and for every $\mathscr{D} \in \mathcal{D}$, there is $C \in \mathscr{D}$ such that $A \subseteq$ Fin $C$.

Here one should think of the context of having some large family $\mathfrak{Y} \in M \prec H_{\lambda}$ for a countable $M, \mathfrak{X}=\mathfrak{Y} \cap M$, and $\mathcal{D}=\{\mathscr{D} \cap M \mid \mathscr{D} \in M$ and $\mathscr{D}$ dense in $\mathfrak{Y} /$ Fin $\}$. We think of $A$ as coming from the large family $\mathfrak{Y}$ and the requirement for $A$ to be $\langle\mathfrak{X}, \mathcal{D}\rangle$-generic is a strengthening of the requirement to be $M$-generic.

Lemma 3.9. Let $\mathfrak{X}$ be a countable family. Assume that $B \in \mathfrak{X} /$ Fin and $G \subseteq \mathfrak{X} /$ Fin is a $V$-generic filter containing $B$. Then in $V[G]$, there is an infinite $A \subseteq \mathbb{N}$ such that $A \subseteq$ Fin $C$ for all $C \in G$. Furthermore, if $\mathcal{D}$ is the collection of dense subsets of $\mathfrak{X} /$ Fin of $V$, then such an $A$ is $\langle\mathfrak{X}, \mathcal{D}\rangle$-generic below $B$.

Proof. Since $G$ is countable and directed in $V[G]$, there exists an infinite $A \subseteq \mathbb{N}$ such that $A \subseteq$ Fin $C$ for all $C \in G$. For the "furthermore" part, fix a dense subset $\mathscr{D}$ of $\mathfrak{X} /$ Fin in $V$. Since there is $C \in G \cap \mathscr{D}$, we have $A \subseteq_{\text {Fin }} C$. It is clear that $A \subseteq_{\text {Fin }} B$ since $B \in G$.

Lemma 3.10. Let $\mathfrak{X}_{0} \subseteq \mathfrak{X}_{1} \subseteq \cdots \subseteq \mathfrak{X}_{\xi} \subseteq \cdots$ for $\xi<\omega_{1}$ be a continuous chain of countable families of reals and let $\mathfrak{X}=\cup_{\xi<\omega_{1}} \mathfrak{X}_{\xi}$. Assume that for every $\xi<\omega_{1}$, if $B \in \mathfrak{X}_{\xi}$ and $\mathcal{D}$ is a countable collection of dense subsets of $\mathfrak{X}_{\xi}$, there is $A \in \mathfrak{X} /$ Fin that is $\left\langle\mathfrak{X}_{\xi}, \mathcal{D}\right\rangle$-generic below $B$. Then $\mathfrak{X}$ is proper.

Proof. Fix a countable $M \prec H_{\lambda}$ such that $\left\langle\mathfrak{X}_{\xi} \mid \xi<\omega_{1}\right\rangle \in M$. It suffices to show that generic conditions exist for such $M$ since these form a club. By Lemma 3.3 $\mathfrak{X} \cap M=\mathfrak{X}_{\alpha}$ where $\alpha=\operatorname{Ord}^{M} \cap \omega_{1}$. Fix $B \in \mathfrak{X}_{\alpha}$ and let $\mathcal{D}=\{\mathscr{D} \cap M \mid \mathscr{D} \in$ $M$ and $\mathscr{D}$ dense in $\mathfrak{X} /$ Fin $\}$. By hypothesis, there is $A \in \mathfrak{X} /$ Fin that is $\left\langle\mathfrak{X}_{\alpha}, \mathcal{D}\right\rangle$ generic below $B$. Clearly $A$ is $M$-generic. Thus, we were able to find an $M$-generic element below every $B \in M \cap \mathfrak{X} /$ Fin.

We are finally ready to show how to force the existence of a proper family of size $\omega_{1}$. 
Theorem 3.11. There is a generic extension of $V$ by a c.c.c. poset that satisfies $\neg \mathrm{CH}$ and contains a proper family of reals of size $\omega_{1}$.

Proof. First, note that we can assume without loss of generality that $V \models \neg \mathrm{CH}$ since this is forceable by a c.c.c. forcing.

The forcing to add a proper family of reals will be a c.c.c. finite support iteration $\mathbb{P}$ of length $\omega_{1}$. The iteration $\mathbb{P}$ will add, step-by-step, a continuous chain $\mathfrak{X}_{0} \subseteq$ $\mathfrak{X}_{1} \subseteq \cdots \subseteq \mathfrak{X}_{\xi} \subseteq \cdots$ for $\xi<\omega_{1}$ of countable arithmetically closed families such that $\cup_{\xi<\omega_{1}} \mathfrak{X}_{\xi}$ will have the property of Lemma 3.10. The idea will be to obtain generic elements for $\mathfrak{X}_{\xi}$, as in Lemma 3.9, by adding generic filters. Once $\mathfrak{X}_{\xi}$ has been constructed, I will force over $\mathfrak{X}_{\xi} /$ Fin below every one of its elements cofinally often before the iteration is over. Every time such a forcing is done, I will obtain a generic element for a new collection of dense sets. This element will be added to $\mathfrak{X}_{\delta+1}$ where $\delta$ is the stage at which the forcing was done.

Fix a bookkeeping function $f$ mapping $\omega_{1}$ onto $\omega_{1} \times \omega$, having the properties that every pair $\langle\alpha, n\rangle$ appears cofinally often in the range and if $f(\xi)=\langle\alpha, n\rangle$, then $\alpha \leq \xi$. Let $\mathfrak{X}_{0}$ be any countable arithmetically closed family and fix an enumeration $\mathfrak{X}_{0}=\left\{B_{0}^{0}, B_{1}^{0}, \ldots, B_{n}^{0} \ldots\right\}$. Each subsequent $\mathfrak{X}_{\xi}$ will be created in $V^{\mathbb{P}_{\xi}}$. Suppose $\lambda$ is a limit and $G_{\lambda}$ is generic for $\mathbb{P}_{\lambda}$. In $V\left[G_{\lambda}\right]$, define $\mathfrak{X}_{\lambda}=\cup_{\xi<\lambda} \mathfrak{X}_{\xi}$ and fix an enumeration $\mathfrak{X}_{\lambda}=\left\{B_{0}^{\lambda}, B_{1}^{\lambda}, \ldots, B_{n}^{\lambda}, \ldots\right\}$. Consult $f(\lambda)=\langle\xi, n\rangle$ and define $\dot{\mathbb{Q}}_{\lambda}=\mathfrak{X}_{\xi} /$ Fin below $B_{n}^{\xi}$. Suppose $\delta=\beta+1$, then $\mathbb{P}_{\delta}=\mathbb{P}_{\beta} * \dot{\mathbb{Q}}_{\beta}$ where $\dot{\mathbb{Q}}_{\beta}$ is $\mathfrak{X}_{\xi} /$ Fin for some $\xi \leq \beta$ below one of its elements. In $V\left[G_{\delta}\right]=V\left[G_{\beta}\right][H]$, let $A \subseteq_{\text {Fin }} B$ for all $B \in H$ and define $\mathfrak{X}_{\delta}$ to be the arithmetic closure of $\mathfrak{X}_{\beta}$ and $A$. Also in $V\left[G_{\delta}\right]$, fix an enumeration $\mathfrak{X}_{\delta}=\left\{B_{0}^{\delta}, B_{1}^{\delta}, \ldots, B_{n}^{\delta}, \ldots\right\}$. Consult $f(\delta)=\langle\xi, n\rangle$ and define $\dot{\mathbb{Q}}_{\delta}=\mathfrak{X}_{\xi} /$ Fin below $B_{n}^{\xi}$. At limits, use finite support.

The poset $\mathbb{P}$ is c.c.c. since it is a finite support iteration of c.c.c. posets (see [?], p. 271). Let $G$ be $V$-generic for $\mathbb{P}$. It should be clear that we can use $G$ in $V[G]$ to construct an arithmetically closed Scott set $\mathfrak{X}=\cup_{\xi<\omega_{1}} \mathfrak{X}_{\xi}$. A standard nice name counting argument shows that $\left(2^{\omega}\right)^{V}=\left(2^{\omega}\right)^{V[G]}$. Since we assumed at the beginning that $V \models \neg \mathrm{CH}$, it follows that $V[G] \models \neg \mathrm{CH}$.

Finally, we must see that $\mathfrak{X}$ satisfies the hypothesis of Lemma 3.10 in $V[G]$. Fix $\mathfrak{X}_{\xi}$, a set $B \in X_{\xi}$, and a countable collection $\mathcal{D}$ of dense subsets of $\mathfrak{X}_{\xi} /$ Fin. Since the poset $\mathbb{P}$ is a finite support c.c.c. iteration and all elements of $\mathcal{D}$ are countable, they must appear at some stage $\alpha$ below $\omega_{1}$. Since we force with $\mathfrak{X}_{\xi} /$ Fin below $B$ cofinally often, we have added a $\left\langle\mathfrak{X}_{\xi}, \mathcal{D}\right\rangle$-generic condition below $B$ at some stage above $\alpha$.

Corollary 3.12. There is a generic extension of $V$ that satisfies $\mathrm{CH}$ and contains a proper family of reals of size $\omega_{1}$ other than $\mathcal{P}(\mathbb{N})$.

Proof. As before, we can assume without loss of generality that $V \models \neg \mathrm{CH}$. Force with $\mathbb{P} * \dot{\mathbb{Q}}$ where $\mathbb{P}$ is the forcing iteration from Theorem 3.11 and $\mathbb{Q}$ is the poset which adds a subset to $\omega_{1}$ with countable conditions. Let $G * H$ be $V$-generic for $\mathbb{P} * \dot{\mathbb{Q}}$, then clearly $\mathrm{CH}$ holds in $V[G][H]$. Also the family $\mathfrak{X}$ created from $G$ remains proper in $V[G][H]$ since $\mathbb{Q}$ is a countably closed forcing, and therefore cannot affect the properness of a family of reals.

We can push this argument further to show that it is consistent with ZFC that there are continuum many proper families of reals of size $\omega_{1}$. 
Theorem 3.13. There is a generic extension of $V$ by a c.c.c. poset that satisfies $\neg \mathrm{CH}$ and contains continuum many proper families of reals of size $\omega_{1}$.

Proof. We start by forcing MA $+\neg \mathrm{CH}$. Since this can be done by a c.c.c. forcing notion ([?], p. 272), we can assume without loss of generality that $V \models \mathrm{MA}+\neg \mathrm{CH}$.

Define a finite support product $\mathbb{Q}=\Pi_{\xi<2^{\omega}} \mathbb{P}^{\xi}$ where every $\mathbb{P}^{\xi}$ is an iteration of length $\omega_{1}$ as described in Theorem 3.11. Since Martin's Axiom implies that finite support products of c.c.c. posets are c.c.c. (see [?], p. 277), the product poset $\mathbb{Q}$ is c.c.c.. Let $G \subseteq \mathbb{Q}$ be $V$-generic, then each $G^{\xi}=G \uparrow \mathbb{P}^{\xi}$ together with $\mathbb{P}^{\xi}$ can be used to build an arithmetically closed family $\mathfrak{X}^{\xi}$ as described in Theorem 3.11 Each such $\mathfrak{X}^{\xi}$ will be the union of an increasing chain of countable arithmetically closed families $\mathfrak{X}_{\gamma}^{\xi}$ for $\gamma<\omega_{1}$. First, I claim that all $\mathfrak{X}^{\xi}$ are distinct. Fixing $\alpha<\beta$, I will show that $\mathfrak{X}^{\alpha} \neq \mathfrak{X}^{\beta}$. Consider $V[G \uparrow \beta+1]=V[G \uparrow \beta]\left[G^{\beta}\right]$ a generic extension by $\left(\mathbb{Q}\lceil\beta) \times \mathbb{P}^{\beta}\right.$. Observe that $\mathfrak{X}^{\alpha}$ already exists in $V[G\lceil\beta]$. Recall that to build $\mathfrak{X}^{\beta}$, we start with an arithmetically closed countable family $\mathfrak{X}_{0}^{\beta}$ and let the first poset in the iteration $\mathbb{P}^{\beta}$ be $\mathfrak{X}_{0}^{\beta} /$ Fin. Let $g$ be the generic filter for $\mathfrak{X}_{0}^{\beta} /$ Fin definable from $G^{\beta}$. The next step in constructing $\mathfrak{X}^{\beta}$ is to pick $A \subseteq \mathbb{N}$ such that $A \subseteq_{\text {Fin }} B$ for all $B \in g$ and define $\mathfrak{X}_{1}^{\beta}$ to be the arithmetic closure of $\mathfrak{X}_{0}^{\beta}$ and $A$. It should be clear that $g$ is definable from $A$ and $\mathfrak{X}_{0}^{\beta}$. Since $g$ is $V[G\lceil\beta]$-generic, it follows that $g \notin V[G \uparrow \beta]$. Thus, $A \notin V[G \uparrow \beta]$, and hence $\mathfrak{X}^{\beta} \neq \mathfrak{X}^{\alpha}$. It remains to show that each $\mathfrak{X}^{\alpha}$ is proper in $V[G]$. Fix $\alpha<2^{\omega}$ and let $V[G]=V\left[G\lceil\alpha]\left[G^{\alpha}\right]\left[G_{\text {tail }}\right]\right.$ where $G_{\text {tail }}$ is the generic for $\mathbb{Q}$ above $\alpha$. By the commutativity of products, $V\left[G\lceil\alpha]\left[G^{\alpha}\right]\left[G_{\text {tail }}\right]=V\left[G\lceil\alpha]\left[G_{\text {tail }}\right]\left[G^{\alpha}\right]\right.\right.$ and $G^{\alpha}$ is $V\left[G\lceil\alpha]\left[G_{\text {tail }}\right]\right.$-generic. Fix a countable $M \prec H_{\lambda}^{V[G]}$ containing the sequence $\left\langle\mathfrak{X}_{\xi}^{\alpha} \mid \xi<\omega_{1}\right\rangle$ as an element. By Lemma 3.3, $M \cap \mathfrak{X}^{\alpha}$ is some $\mathfrak{X}_{\gamma}^{\alpha}$. This is the key step of the proof since it allows us to know exactly what $M \cap \mathfrak{X}^{\alpha}$ is, even though we know nothing about $M$. Let $G_{\xi}^{\alpha}=G^{\alpha} \uparrow \mathbb{P}_{\xi}^{\alpha}$ for $\xi<\omega_{1}$. Let $\mathcal{D}=\left\{\mathscr{D} \cap M \mid \mathscr{D} \in M\right.$ and $\mathscr{D}$ dense in $\mathfrak{X}^{\alpha} /$ Fin $\}$. There must be some $\beta<\omega_{1}$ such that $\mathcal{D} \in V\left[G\lceil\alpha]\left[G_{\text {tail }}\right]\left[G_{\beta}^{\alpha}\right]\right.$. By construction, there must be some stage $\delta>\beta$ at which we forced with $\mathfrak{X}_{\gamma}^{\alpha} /$ Fin and added a set $A$ such that $A \subseteq_{\text {Fin }} B$ for all $B \in H$ where $G_{\delta+1}^{\alpha}=G_{\delta}^{\alpha} * H$. Now observe that $H$ is $V\left[G\lceil\alpha]\left[G_{\text {tail }}\right]\left[G_{\delta}^{\alpha}\right]\right.$-generic for $\mathfrak{X}_{\gamma}^{\alpha} /$ Fin. Therefore $H$ meets all the sets in $\mathcal{D}$. So we can conclude that $A$ is $M$-generic.

A standard nice name counting argument will again show that $\left(2^{\omega}\right)^{V}=\left(2^{\omega}\right)^{V[G]}$. Thus, $V[G]$ satisfies $\neg \mathrm{CH}$ and contains continuum many proper families of reals of size $\omega_{1}$.

Similar techniques allow us to force the existence of a piecewise proper family of reals of size $\omega_{2}$.

Lemma 3.14. Let $\mathfrak{X}_{0} \subseteq \mathfrak{X}_{1} \subseteq \cdots \subseteq \mathfrak{X}_{\xi} \subseteq \cdots$ for $\xi<\omega_{1}$ be a continuous chain of countable families of reals and let $\mathfrak{X}=\cup_{\xi<\omega_{1}} \mathfrak{X}_{\xi}$. Assume that for every $\xi<\omega_{1}$, if $B \in \mathfrak{X}_{\xi}$ and $\mathcal{D}$ is a countable collection of dense subsets of $\mathfrak{X}_{\xi}$, there is $A \in \mathfrak{X} /$ Fin that is $\left\langle\mathfrak{X}_{\xi}, \mathcal{D}\right\rangle$-generic below $B$. Then $\mathfrak{X}$ is proper and $\mathfrak{X}$ remains proper after forcing with any absolutely c.c.c. poset.

Proof. The proof is a straightforward modification of the proof of Theorem 3.5 Let $\mathbb{P}$ be an absolutely c.c.c. poset and $g \subseteq \mathbb{P}$ be $V$-generic. We need to show that $\mathfrak{X}$ is proper in $V[g]$. Fix a countable $M[g] \prec H_{\lambda}[g]$ in $V[g]$ such that $\left\langle\mathfrak{X}_{\xi}\right.$ : $\left.\xi<\omega_{1}\right\rangle, \mathbb{P} \in M[g]$ and $M \subseteq V$. Let $\mathfrak{X}_{\alpha}=M \cap \mathfrak{X}$ and let $\mathcal{D}=\left\{\mathscr{D} \cap \mathfrak{X}_{\xi} \mid \mathscr{D} \in\right.$ 
$M$ and $\mathscr{D}$ dense in $\mathfrak{X}\}$. Observe that $\mathcal{D} \subseteq V$ and $|\mathcal{D}|=\omega$. Define $\mathcal{E}$ as in proof of Theorem 3.5. Now choose $A \in \mathfrak{X}$ that is $\left\langle\mathfrak{X}_{\alpha}, \mathcal{E}\right\rangle$-generic in $V$. It follows that $A$ is $M$-generic. Next proceed exactly as in the proof of Theorem 3.5. using the fact that $\mathbb{P}$ is absolutely c.c.c. in the final stage of the argument.

Theorem 3.15. There is a generic extension of $V$ by a c.c.c. poset which contains a piecewise proper family of reals of size $\omega_{2}$.

Proof. We will define a c.c.c. forcing iteration $\mathbb{Q}_{\omega_{2}}$ of length $\omega_{2}$ to accomplish this. Let $\mathbb{Q}_{0}$ be the forcing to add a proper family $\mathfrak{X}_{0}$ of size $\omega_{1}$ (Theorem 3.11). At the $\alpha^{\text {th }}$-stage, force with the poset to add a proper family $\mathfrak{X}_{\alpha} \supseteq \cup_{\beta<\alpha} \mathfrak{X}_{\beta}$. Observe here, that the poset from Theorem 3.11 can be very easily modified to the poset which adds a proper family extending any family of reals from the ground model. Let $G \subseteq \mathbb{Q}_{\omega_{2}}$ be $V$-generic. I claim each $\mathfrak{X}_{\alpha}$ remains proper in $V[G]$. Fix $\mathfrak{X}_{\alpha}$ and factor the forcing $\mathbb{Q}_{\omega_{2}}=\mathbb{Q}_{\alpha} * \mathbb{Q}_{\text {tail }}$. The family $\mathfrak{X}_{\alpha}$ is proper in $V\left[G_{\alpha}\right]$ and $\mathbb{Q}_{\text {tail }}$ is absolutely c.c.c. in $V\left[G_{\alpha}\right]$. The poset $\mathbb{Q}_{\text {tail }}$ is absolutely c.c.c since the forcing to add a proper family is a finite support iteration of countable posets. Thus, by Lemma 3.14. $\mathfrak{X}_{\alpha}$ remains proper in $V\left[G_{\alpha}\right]\left[G_{\text {tail }}\right]$. Thus, $\mathfrak{X}=\cup_{\alpha<\omega_{2}} \mathfrak{X}_{\alpha}$ is clearly piecewise proper.

By exactly following the proof of Theorem 3.13, we can extend Theorem 3.15 to obtain:

Theorem 3.16. There is a generic extension of $V$ by a c.c.c. poset which contains continuum many piecewise proper families of reals of size $\omega_{2}$.

By Enayat's [?] example of a non-proper arithmetically closed family of size $\omega_{1}$, we know that there are piecewise proper families that are not proper. This follows by recalling that arithmetically closed families of size $\omega_{1}$ are trivially piecewise proper. It is not clear whether every proper family has to be piecewise proper. In particular, it is not known whether $\mathcal{P}(\mathbb{N})$ is piecewise proper. It follows that $\mathcal{P}(\mathbb{N})$ can be piecewise proper from the proof of Theorem 3.15 since we can modify the construction to end up with $\mathfrak{X}=\mathcal{P}(\mathbb{N})$.

Finally, I will discuss a possible construction for proper families under PFA. The idea is, in some sense, to mimic the forcing iteration like that of Theorem 3.11 in the ground model. Unfortunately, the main problem with the construction is that it is not clear whether we are getting the whole $\mathcal{P}(\mathbb{N})$. This problem never arose in the forcing construction since we were building families of size $\omega_{1}$ and knew that the continuum was larger than $\omega_{1}$. I will describe the construction and a possible way of ensuring that the resulting family is not $\mathcal{P}(\mathbb{N})$.

Fix an enumeration $\left\{\left\langle A_{\xi}, B_{\xi}\right\rangle \mid \xi<\omega_{2}\right\}$ of $\mathcal{P}(\omega) \times \mathcal{P}(\omega)$. Also fix a bookkeeping function $f$ from $\omega_{2}$ onto $\omega_{2}$ such that each element appears cofinally in the range. I will build a family $\mathfrak{X}$ of size $\omega_{2}$ as the union of an increasing chain of arithmetically closed families $\mathfrak{X}_{\xi}$ for $\xi<\omega_{2}$. Start with any arithmetically closed family $\mathfrak{X}_{0}$ of size $\omega_{1}$. Suppose we have constructed $\mathfrak{X}_{\beta}$ for $\beta \leq \alpha$ and we need to construct $\mathfrak{X}_{\alpha+1}$. Consult $f(\alpha)=\gamma$ and consider the pair $\left\langle A_{\gamma}, B_{\gamma}\right\rangle$ in the enumeration of $\mathcal{P}(\omega) \times \mathcal{P}(\omega)$. First, suppose that $A_{\gamma}$ codes a countable family $\mathfrak{Y} \subseteq \mathfrak{X}_{\alpha}$ and $B_{\gamma}$ codes a countable collection $\mathcal{D}$ of dense subsets of $\mathfrak{Y}$. Let $G$ be some filter on $\mathfrak{Y}$ meeting all sets in $\mathcal{D}$ and let $A \subseteq \subseteq_{\text {Fin }} C$ for all $C \in G$. Define $\mathfrak{X}_{\alpha+1}$ to be the arithmetic closure of $\mathfrak{X}_{\alpha}$ and $A$. If the pair $\left\langle A_{\gamma}, B_{\gamma}\right\rangle$ does not code such information, let $\mathfrak{X}_{\alpha+1}=\mathfrak{X}_{\alpha}$. At limit stages take unions. 
I claim that $\mathfrak{X}$ is proper. Fix some countable $M \prec H_{\lambda}$ containing $\mathfrak{X}$. Let $\mathfrak{Y}=M \cap \mathfrak{X}$ and let $\mathcal{D}=\{\mathscr{D} \cap M \mid \mathscr{D} \in M$ and $\mathscr{D}$ dense in $\mathfrak{X} /$ Fin $\}$. There must be some $\gamma$ such that $\left\langle A_{\gamma}, B_{\gamma}\right\rangle$ codes $\mathfrak{Y}$ and $\mathcal{D}$. Let $\delta$ such that $M \cap \mathfrak{X}$ is contained in $\mathfrak{X}_{\delta}$, then there must be some $\alpha>\delta$ such that $f(\alpha)=\gamma$. Thus, at stage $\alpha$ in the construction we considered the pair $\left\langle A_{\gamma}, B_{\gamma}\right\rangle$. Since $\alpha>\delta$, we have $M \cap \mathfrak{X}=M \cap \mathfrak{X}_{\alpha}$. It follows that at stage $\alpha$ we added an $M$-generic set $A$ to $\mathfrak{X}$.

A way to prove that $\mathfrak{X} \neq \mathcal{P}(\mathbb{N})$ would be to show that some fixed set $C$ is not in $\mathfrak{X}$. Suppose the following question had a positive answer:

Question 3.17. Let $\mathfrak{X}$ be an arithmetically closed family such that $C \notin \mathfrak{X}$ and $\mathfrak{Y} \subseteq \mathfrak{X}$ be a countable family. Is there a $\mathfrak{Y} /$ Fin-name $\dot{A}$ such that $1_{\mathfrak{Y} / \text { Fin }} \Vdash " \dot{A} \subseteq$ Fin $B$ for all $B \in \dot{G}$ and $\check{C}$ is not in the arithmetic closure of $\dot{A}$ and $\check{\mathfrak{X}} "$ ?

Assuming that the answer to Question 3.17 is positive, let us construct a proper family $\mathfrak{X}$ in such a way that $C$ is not in $\mathfrak{X}$. We will carry out the above construction being careful in our choice of the filters $G$ and elements $A$. Start with $\mathfrak{X}_{0}$ that does not contain $C$ and assume that $C \notin \mathfrak{X}_{\alpha}$. Suppose the pair $\left\langle A_{\gamma}, B_{\gamma}\right\rangle$ considered at stage $\alpha$ codes meaningful information. That is, $A_{\gamma}$ codes a countable family $\mathfrak{Y} \subseteq \mathfrak{X}_{\alpha}$ and $B_{\gamma}$ codes a countable collection $\mathcal{D}$ of dense subsets of $\mathfrak{Y}$. Choose some transitive $N \prec H_{\omega_{2}}$ of size $\omega_{1}$ such that $\mathfrak{X}_{\alpha}, \mathfrak{Y}$, and $\mathcal{D}$ are elements of $N$. Since we assumed a positive answer to Question 3.17, $H_{\omega_{2}}$ satisfies that there exists a $\mathfrak{Y} /$ Fin-name $\dot{A}$ such that $1_{\mathfrak{Y} / \text { Fin }} \Vdash " \dot{A} \subseteq_{\text {Fin }} B$ for all $B \in \dot{G}$ and $\check{C}$ is not in the arithmetic closure of $\dot{A}$ and $\mathfrak{X}_{\alpha}$ ". But then $N$ satisfies the same statement by elementarity. Hence there is $\dot{A} \in N$ such that $N$ satisfies $1_{\mathfrak{Y} / \text { Fin }} \Vdash " \dot{A} \subseteq_{\text {Fin }} B$ for all $B \in \dot{G}$ and $\check{C}$ is not in the arithmetic closure of $\dot{A}$ and $\check{\mathfrak{X}}_{\alpha}$ ". Now use PFA to find an $N$-generic filter $G$ for $\mathfrak{Y} /$ Fin. Since $G$ is fully generic for the model $N$, the model $N[G]$ will satisfy that $C$ is not in the arithmetic closure of $\mathfrak{X}_{\alpha}$ and $A=\dot{A}_{G}$. Thus, it is really true that $C$ is not in the arithmetic closure of $\mathfrak{X}_{\alpha}$ and $A$. Since $G$ also met all the dense sets in $\mathcal{D}$ and $A \subseteq_{\text {Fin }} B$ for all $B \in G$, we can let $\mathfrak{X}_{\alpha+1}$ be the arithmetic closure of $\mathfrak{X}_{\alpha}$ and $A$. Thus, $C \notin \mathfrak{X}_{\alpha+1}$. We can conclude that $C \notin \mathfrak{X}$.

\section{Questions}

Question 4.1. Can ZFC or ZFC + PFA prove the existence of an uncountable proper family of reals other than $\mathcal{P}(\mathbb{N})$ ?

Question 4.2. Can ZFC or ZFC + PFA prove the existence of a piecewise proper family of size $\omega_{2}$ ?

Question 4.3. Is it consistent with ZFC that there are proper families of reals of size $\omega_{2}$ other than $\mathcal{P}(\mathbb{N})$ ?

Question 4.4. What is the answer to Question 3.17?

Question 4.5. Can $\mathcal{P}(\mathbb{N})$ be non-piecewise proper?

New York City College of Technology (CUNy), Mathematics, 300 Jay Street, BrookLYN, NY 11201 USA

E-mail address: vgitman@nylogic.org 